
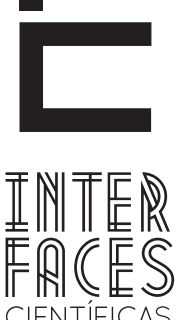

CIENTÍFICAS

HUMANASE SOCIAIS

ISSN IMPRESSO 2316-3348

E-ISSN 2316-3801

DOI - 10.17564/2316-3801.2016v5n2p97-112

\title{
NETWORKING NAS RELAÇÕES INSTITUCIONAIS: UMA ANÁLISE NO CONGRESSO ANPCONT ENTRE O PERÍODO DE 2013 A 2015
}

NETWORKING IN INSTITUTIONAL RELATIONS: AN ANALYSIS OF THE ANPCONT CONFERENCE FROM 2013 TO 2015

NETWORKING EN RELACIONES INSTITUCIONALES: UN ANÁLISIS EN EL CONGRESO ANPCONT ENTRE EL PERÍODO 20132015

\section{RESUMO}

A expansão nos estudos de redes sociais vem aumentando nos últimos anos, cientistas sociais, pesquisadores e acadêmicos se questionam sobre a capacidade e o poder do chamado networking nas relações entre os atores de uma rede e seus benefícios. Este estudo teve como objetivo verificar quais as redes sociais institucionais formadas a partir da produção científica do Congresso ANPCONT. A população da pesquisa abrangeu 334 artigos publicados nesse congresso entre o período de 2013 a 2015. A amostra final foi composta de 165 artigos, com 17 produções individuais e 148 coautorias em grupos interligados na produção científica. Os testes foram realizados por meio do software Sociais Network Analysis UCINET 6.531, Netdraw- Network Visualization Program 2.153 e Microsoft Office
Excel 2010. A análise demonstrou que as instituições mais profícuas foram USP, UNISINOS, UFPR e FURB. Conclui-se nesse estudo que a rede de cooperação e produção científica entre as instituições apresentam ligações fracas e conexões esparsas. No entanto, constatou-se a formação de uma rede de cooperação com laços centrais "entrada/saída" das quais se destacam, além das citadas, as instituições: MACKENZIE, UFRJ, UFMG, UFU e UFGV, UnB/UFPB/UFRN e FGV.

\section{PALAVRAS-CHAVE}

Redes Sociais. Cooperação. Produção Científica. 


\section{ABSTRACT}

The number of studies on social networks has increased in recent years. Social scientists, researchers, and scholars are questioning the ability and power of so-called networking in relationships between the actors of a network and the benefits of these relationships. This study aims to determine which institutional social networks formed as a result of the scientific production of the ANPCONT Conference. The research population included 334 articles published at the conference from 2013 to 2015. The final sample comprised 165 articles with 17 individual productions and 148 co-authorships in interconnected groups in scientific production. Tests were conducted using the Social Network Analysis software UCINET 6.531, Netdraw-Network Visualization Program 2.153, and Microsoft Office Excel
2010. The analysis shows that the more productive institutions were USP, UNISINOS, UFPR, and FURB. This study concludes that the network of cooperation and scientific production among institutions has weak links and sparse connections. However, the formation of a network of cooperation with "input/ output" central links was evident, among which, besides the aforementioned, the following institutions stood out: MACKENZIE, UFRJ, UFMG, UFU and UFGV, UNB/UFPB/ UFRN, and FGV.

\section{KEYWORDS}

Social networks. Cooperation. Scientific production.

\section{RESUMEN}

La expansión en los estudios de las redes sociales ha aumentado en los últimos años. Los científicos sociales, investigadores y estudiosos se preguntan sobre la capacidad y el poder de la "creación de redes" en las relaciones entre los actores de una red y sus beneficios. Este estudio tuvo como objetivo determinar qué redes sociales institucionales se formaron desde la producción científica del Congreso ANPCONT. La población de la investigación incluyó 334 artículos publicados en el Congreso entre el período de 2013 a 2015. La muestra final consta de 165 Artículo y 17 producciones individuales y 148 co-autorías de grupos interconectados en la producción científica. Las pruebas se llevaron a cabo por el UCINET software de análisis de redes sociales 6531, Netdraw- Red programa de visualización 2153 y Microsoft Office Excel 2010. El análisis mostró que las instituciones eran más fructíferas en USP, UNISINOS, UFPR y FURB. Se concluye en este estudio que la red de cooperación y producción científica entre las instituciones tienen vínculos débiles y conexiones dispersas. Sin embargo, no fue la formación de una red de cooperación con eslabones centrales "entrada / salida" de los cuales se destacan, además de lo anterior, las instituciones: Mackenzie, UFRJ, UFMG, UFU y UFGV, UNB / UFPB / UFRN y FGV.

\section{PALABRAS CLAVE}

Redes sociales. Cooperación. Producción científica. 


\section{INTRODUÇ̄̃̃O}

As pesquisas que envolvem as análises de redes sociais vêm crescendo ao longo do tempo entre pesquisadores de diversas áreas do conhecimento na comunidade acadêmica. Os elementos que compõem estas ligações em redes e laços que caracterizam as estruturas de conexões entre os partícipes são definidos por nós. Nesse contexto, os atores são ligados e descrevem as relações entre os membros de um grupo e não apenas os seus atributos (HANNEMAN; RIDDLE, 2005).

O conceito de redes sociais vem sendo analisado desde a revolução industrial, sendo um tema inquietante na comunidade científica. Na atualidade, ele tem sugerido enfoques e vertentes diversos, aproximando pesquisadores das mais diversas áreas do conhecimento (BRUFEN; GABRIEL JR; SORRIBAS, 2011). Balestrin, Verschoore e Reyes (2010), destacam que no contexto acadêmico os estudos das redes sociais têm evoluído substancialmente nos últimos anos.

Para Silva e outros autores (2006) o crescimento ocorre devido ao aumento de dados disponíveis para análise, avanço do poder computacional, bem como a ampliação de áreas do conhecimento que utilizam as redes sociais como instrumento para análises. As redes sociais segundo Nascimento e Beuren (2011), são ligações oriundas das redes de relacionamentos, estabelecidas pelos atores, que podem fortalecer e consolidar as ideias, atividades de pesquisa e de produção científica em conjunto.

O sociólogo J. A. Barnes em 1954 passou a utilizar o termo rede social para indicar padrões de relacionamentos entre grupos. 0 termo rede associa-se ao "social" para especificar o campo, mas não delimita uma área específica, pois o mesmo é utilizado em várias áreas do conhecimento, como por exemplo: Antropologia, Sociologia, Economia, Ciências Políticas, Ciências da Informação e Ciências da Comunicação. No Brasil as primeiras evidências de Análises de Redes
Sociais (ARS) foram de autoria de Reed Nelson em 1984, onde o autor discute questões metodológicas que abordam a modelação de blocos sociais nos estudos de estruturas organizacionais (NELSON, 1984; MARTELETO, 2010).

Os programas de pós-graduação contribuíram significativamente para evolução, construção e disseminação do conhecimento sobre estudos que utilizam o método de análise de redes sociais (NASCIMENTO; BEUREN, 2011). Nesse contexto, a utilização de redes na análise da produção científica permite a observação de aspectos interdisciplinares decorrentes da colaboração entre os pesquisadores, o que proporciona a estruturação de um dado campo do conhecimento.

Maciel (2007) faz referência a importância de analisar redes sociais como subsídio para entender os relacionamentos e a dinâmica interorganizacional. As ligações provenientes, tanto de dependência como de interdependência dos pesquisadores de redes, vem se prevalecendo pelo interesse em trocar informações nas configurações desses grupos. Dessa forma, a questão de pesquisa desse estudo busca saber quais as redes sociais institucionais são formadas por meio da produção científica entre pesquisadores?

0 estudo teve como objetivo verificar quais as redes sociais institucionais são formadas nos estudos apresentados no Congresso ANPCONT - promovido anualmente pela Associação Nacional de Programas de Pós-Graduação em Ciências Contábeis (ANPCONT) - no período entre 2013 a 2015.

Nesta concepção, este estudo insere-se no contexto das pesquisas que visam entender as redes sociais no âmbito da produção científica, buscando quantificar a troca de informações e a construção do conhecimento científico no âmbito das ciências sociais aplicadas. Na estruturação apresenta-se uma breve 
introdução e no referencial aborda-se a teoria de grafos e redes sociais, os sistemas de redes sociais. $\mathrm{Na}$ sequência, os procedimentos metodológicos, resultado e discussões, seguidos pelas considerações finais e referências.

\section{A TEORIA DE GRAFOS E REDES SOCIAIS}

Nesta seção apresentam-se os aspectos que embasaram teoricamente este estudo. Primeiramente elencam-se aspectos relacionados à teoria de grafos e redes sociais e logo após sobre o Sistema de Redes.

A análise de rede social pode ser aplicada em diversas áreas do conhecimento, o uso desse método foi desenvolvido sobre múltiplas influências. As mais importantes contribuições são atribuídas à matemática e a ciência da computação por meio da teoria de grafos. Na sociologia, as contribuições nas relações de redes podem ser descritas como sendo provenientes da sociometria, sob a rubrica de importantes autores como: Lewin e Moreno, e também da escola antropológica com os autores W. Lloyd Warner da Harvard School, e Barnes, Mitchell e Bott da Manchester (OTTE; ROUSSEAU, 2002).

Os primórdios da teoria de redes encontram-se nos trabalhos do matemático Ëuler que criou o teorema da teoria dos grafos. Nesta teoria, um grafo é uma reprodução de um conjunto de nós interligados por arestas que, em conjunto, formam uma rede. A percepção das coisas como uma rede seria crucial para a compreensão das relações complexas do mundo a nossa volta (RECUERO, 2005). A partir das ideias de Ëuler, vários pesquisadores dedicaram-se a entender quais eram as propriedades dos grafos e como se dava o processo de sua construção (BUCHANAN, 2002; BARABÁSI, 2003; WATTS, 2003).

Segundo Loiola e Moura (1996) as relações subjacentes às análises de redes sociais vinculam-se aos estudos efetuados no campo dos movimentos sociais que, correspondem às articulações/interações entre organizações e indivíduos. A interação entre atores de uma rede parece funcionar como uma estratégia, na tentativa de ampliar o número de parceiros e sua diversidade, considerando a viabilização de projetos de interesses comuns. As duas principais características básicas observadas são: a interação de atores e/ou organizações formais ou informais, e a regularidade nessas interações.

A teoria dos grafos, na sociologia, é uma das bases do estudo das redes sociais, ancorada na chamada análise estrutural de Degenne e Forsé (1999), proveniente das décadas de 1960 e 1970, que dedica especial atenção à análise das estruturas sociais. Para Bastos e Santos (2007) a teoria dos grafos, amparada por um corpo de axiomas matemáticos e fórmulas, constitui um recurso teórico e metodológico útil para os estudos que tem como enfoque os sistemas organizacionais, constituídos por relações e conexões existentes entre os membros de uma rede social.

Lozares (1996) ressalta que, a ideia principal na análise de redes sociais, centra-se no pressuposto de como as pessoas sentem, pensam e se manifestam em padrões de relacionamentos estruturais, opondo-se à ideia de que os atributos individuais são a causa desses padrões comportamentais.

A teoria sobre social network é utilizada para estudar como os laços sociais entre os atores de uma determinada rede podem afetar o desempenho da organização. Para Granovetter (1981), uma das questões clássicas da teoria social relaciona-se a como o comportamento e as instituições são afetadas pelas relações sociais, analisando até que ponto a ação econômica está imersa nas estruturas das relações sociais no âmbito da moderna sociedade industrial. Burt (1992) observa que diferentes posições dentro de uma rede de relacionamento afetam as oportunidades dos atores. As relações estruturais podem ter efeitos distintos entre pessoas de diferentes atributos 
ou organizações de diferentes tipos. Entretanto, estes efeitos ocorrerem porque os atributos e formas de organização estão correlacionados com as diferentes posições na estrutura social.

\section{SISTEMAS DE REDES SOCIAIS}

As redes sociais referem-se a uma estrutura composta por atores, interações ou laços, que decorrem de algum tipo de relação entre dois ou mais atores, onde os atores são representados como nós e as interações entre eles, por meio de arcos que conectam os nós (WASSERMAN; FAST, 1994; DEGENNE; FORSE, 1999).

Pinto e Junqueira (2009) definem as redes sociais como um conjunto de indivíduos e organizações conectados que vão construindo e reconstruindo a estrutura social. Essa vinculação se dá por meio das relações sociais que são manifestadas de modos diversos e expressam a complexidade do mundo social. Hakanson (1987) aborda redes sociais como um conjunto de elementos ligados por meio de um conjunto de relações específicas, onde as redes são estruturadas a partir da definição dos papéis, atribuições e relações entre seus atores.

Segundo Degenne e Forsé (1999) a análise das redes sociais parte de duas grandes visões do objeto de estudo, primeiro as redes inteiras (whole networks) e segundo as redes personalizadas (ego-centered networks). As redes inteiras são focadas nas relações estruturais com o grupo social. Nessa visão, as redes são assinaturas da identidade social, ou seja, como o padrão de relações entre os indivíduos estão mapeando as preferências e características dos próprios envolvidos na rede (WATTS, 2003). As redes personalizadas possuem o foco no papel social de como um indivíduo poderia ser compreendido não apenas por meio das redes a que pertence, mas igualmente, por meio das posições que tem dentro dessas redes.
As redes são sistemas compostos por nós e conexões, representados por indivíduos, grupos, organizações etc., conectados por algum tipo de relação (SILVA ET AL., 2006). Segundo Martins (2009), as redes sociais são um conjunto de contatos que ligam vários atores de diferentes formas de rede de relacionamento, estabelecida pelo indivíduo e/ou organizações, dentro e fora de um determinado ambiente analisado.

Britto (2002) apresenta os quatro elementos morfológicos que compõem a análise de redes sociais: os nós, as posições, as ligações e os fluxos (Figura 1).

Figura 1 - Elementos morfológicos nas análises de Redes

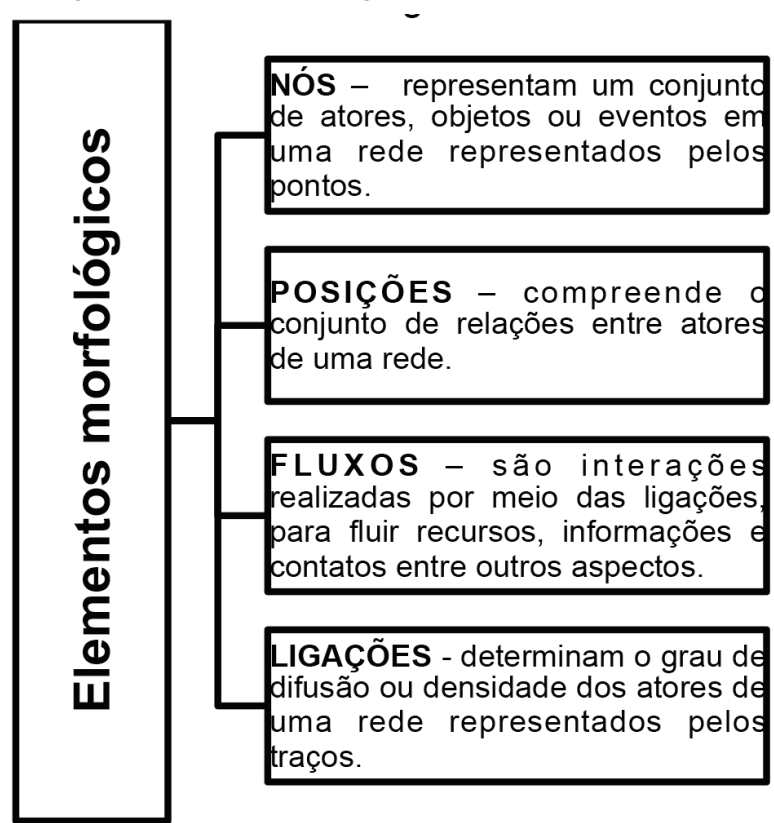

Fonte: Adaptado de Britto (2002).

No que tange a interatividade nos estudos de redes sociais, Rossoni, Hocayen-da-Silva e Junior (2008) proferem que, as relações nas análises de redes, centram-se em estimar a existência de laços entre atores e sua reciprocidade. 0 nível de díade basicamente busca avaliar a probabilidades de relacionamento entre pares de atores, enquanto a tríade 
foca sua análise entre três atores e as relações estabelecida entre os membros dessa rede.

Os laços relacionais representam a coleção de um tipo específico de laço entre os membros de um grupo ou equipe, que é representado pelas relações entre os atores (WASSERMAN; FAST, 1994). Estes laços ou ligações formam uma densa malha de relacionamento, e muitos dos possíveis laços relacionais estão presentes. Entretanto, os laços fracos, são os menos suscetíveis a serem socialmente envolvidos numa rede social de baixa densidade, ou seja, uma rede em que muitas das possíveis linhas relacionais estão ausentes (GRANOVETTER, 1983).

\section{PROCEDIMENTOS METODOLÓGICOS}

Análise de redes sociais é uma metodologia poderosa para descrever e analisar as inter-relações das unidades ou nós dentro de uma rede. Os procedimentos utilizados para estudar os fenômenos estruturais de uma rede podem ser utilizados em níveis organizacionais e interorganizacionais. No nível organizacional, a rede analisa as relações entre indivíduos ou grupos dentro da empresa, enquanto no nível interorganizacional, a análise de redes sociais centra-se nas inter-relações das organizações em redes horizontais e verticais (CARTER; ELLRAM; TATE, 2007).

Esta pesquisa caracteriza-se como descritiva, por meio da identificação das redes sociais na produção cientifica do congresso ANPCONT. Em relação ao objetivo da pesquisa é caracterizada como documental, tendo em vista a análise dos artigos científicos, para o estudo bibliométrico.

A pesquisa documental tem como principal característica o levantamento de material editado, tais como livros, periódicos, entre outros (MARTINS; THEÓPHILO, 2007). Já as pesquisas biblimétricas na visão de Macia-Chapula (1998), abrangem o estudo dos as- pectos quantitativos da produção, disseminação e uso da informação registrada.

A população da pesquisa compreendeu 334 artigos publicados no congresso ANPCONT no período de 2013 a 2015. No entanto, o critério para seleção da amostra foi o número de instituições que publicaram no congresso nesse período e o número de interações entre estas instituições. As relações sociais foram estabelecidas por 83 publicações entre as instituições, com 17 produções individuais e a inserção de grupos interconectados por meio de 148 coautorias, totalizando 165 artigos para amostra final.

Os testes para verificar as relações entre os atores foram realizadas por meio do software Sociais $\mathrm{Ne}$ twork Analysis - UCINET 6.531, Netdraw - Network Visualization Program 2.153 e Microsoft Office Excel 2010. Os cálculos dos indicadores da rede social consideraram a matriz de relações dos 165 artigos em uma planilha constituídas pelas células xy. Neste caso, atribuindo o valor 1a existência de uma ou mais interações e zero no caso de ausência, para filas e colunas que cada nó apresenta com o restante dos indivíduos membros dessa rede.

\section{RESULTADO E DISCUSSÃO}

A análise de redes, contemplada com teorias e conceitos é considerada uma alternativa metodológica que admite uma interlocução entre as ciências sociais e ciências da informação. 0 estudo nas relações de uma rede social permite traçar os fluxos de informações e as construções cognitivas e simbólicas de atores desse grupo. A abordagem metáfora sobre redes sociais teve seus registros iniciais na sociologia e depois na psicologia e na antropologia, para associar o comportamento individual dos atores de um grupo à estrutura a qual ele pertence. 0 estudo de rede é considerado uma metodologia chamada de sociometria, cujo instrumento de análise apresenta- 
-se na forma de um sociograma, isto é, diagramas de redes que permitem a representação visual da estrutura que está sendo considerada (MARTELETO, 2001; MARTELETO; SILVA, 2004).

Nessa perspectiva, a abordagem de rede permite aos analistas estudar um conjunto mais diversificado de fenômenos estruturais, tais como: A densidade de agrupamento de uma rede; verificar se os laços da rede são estritamente especializados ou amplamente multiplex; verificar as variações, restrições quanto ao tamanho e heterogeneidade, bem como os laços e as posições estruturais indireta afetam o comportamen- to dos indivíduos (WELLMAN, 1999). A Tabela 1 apresenta o número de artigos publicados e interações entre as instituições e os respectivos colaboradores que compõem a amostra, totalizando 165 ocorrências.

A Universidade de São Paulo (USP) apresentou uma maior interação em coautorias, 10\% levando em consideração a frequência em número de artigos publicados com outras instituições. Da mesma forma, UNISINOS, UFPR e FURB apresentaram resultados significativos, ambas com $5 \%$, no número de colaborações entre os autores, resultado que sugere a aparente centralidade de algumas instituições.

\begin{tabular}{|c|c|c|c|c|c|c|c|}
\hline INSTITUIÇÃO & $\mathrm{F}$ & $\%$ & INSTITUIÇÃO & $\mathrm{F}$ & INSTITUIÇÃO & $\mathrm{F}$ & $\%$ \\
\hline USP & 16 & 10 & UFSM & 02 & PUCPR & 01 & 01 \\
\hline UNISINOS & 09 & 05 & UNIPAMPA & 02 & ESADE & 01 & 01 \\
\hline UFPB & 07 & 04 & ESTACIO & 02 & ISPA & 01 & 01 \\
\hline UFPR & 08 & 05 & CEFET/MG & 01 & FAED & 01 & 01 \\
\hline FURB & 08 & 05 & UNEB & 01 & UFERSA & 01 & 01 \\
\hline MACKENZIE & 05 & 03 & UNIFOR & 01 & IBMEC & 01 & 01 \\
\hline UFRJ & 05 & 03 & UCB & 01 & IMED & 01 & 01 \\
\hline UnB/UFPB/UFRN & 06 & 04 & ITCP & 01 & UARK & 01 & 01 \\
\hline UFMG & 05 & 03 & UFF & 01 & UEMS & 01 & 01 \\
\hline UFU & 04 & 02 & UFPE & 01 & UDESC & 01 & 01 \\
\hline FGV & 06 & 04 & FAESA & 01 & U.S.T. & 00 & 00 \\
\hline UERJ & 03 & 02 & U.P.P. & 01 & U.V. & 00 & 00 \\
\hline UFRGS & 02 & 01 & FURG & 01 & FECAP & 00 & 00 \\
\hline UFBA & 02 & 01 & FAMETRO & 01 & UEPB & 00 & 00 \\
\hline UFSC & 04 & 02 & UEM & 01 & PUC MINAS & 00 & 00 \\
\hline FUCAPE & 04 & 02 & USCS & 01 & UNIFAVIP & 00 & 00 \\
\hline UNIVALI & 02 & 01 & F.N.H & 01 & PUC - CAMP. & 00 & 00 \\
\hline FAT & 02 & 01 & UNEMAT & 01 & UFAM & 00 & 00 \\
\hline
\end{tabular}




\begin{tabular}{llllllll}
\hline \multicolumn{1}{c}{ INSTITUIÇÃO } & $F$ & $\%$ & \multicolumn{1}{c}{ INSTITUIÇÃO } & $F$ & INSTITUIÇÃO & $F$ & $\%$ \\
\hline UNIFRA & 02 & 01 & UFRRJ & 01 & PUCRJ & 00 & 00 \\
USP & 16 & 10 & UFSM & 02 & PUCPR & 01 & 01 \\
UNISINOS & 09 & 05 & UNIPAMPA & 02 & ESADE & 01 & 01 \\
UFPB & 07 & 04 & ESTACIO & 02 & ISPA & 01 & 01 \\
UFPR & 08 & 05 & CEFET/MG & 01 & FAED & 01 & 01 \\
FURB & 08 & 05 & UNEB & 01 & UFERSA & 01 & 01 \\
MACKENZIE & 05 & 03 & UNIFOR & 01 & IBMEC & 01 & 01 \\
UFRJ & 05 & 03 & UCB & 01 & IMED & 01 & 01 \\
UnB/UFPB/UFRN & 06 & 04 & ITCP & 01 & UARK & 01 & 01 \\
UFMG & 05 & 03 & UFF & 01 & UEMS & 01 & 01 \\
UFU & 04 & 02 & UFPE & 01 & UDESC & 01 & 01 \\
FGV & 06 & 04 & FAESA & 01 & U.S.T. & 00 & 00 \\
UERJ & 03 & 02 & U.P.P. & 01 & U.V. & 00 & 00 \\
UFRGS & 02 & 01 & FURG & 01 & FECAP & 00 & 00 \\
UFBA & 02 & 01 & FAMETRO & 01 & UEPB & 00 & 00 \\
UFSC & 04 & 02 & UEM & 01 & PUC MINAS & 00 & 00 \\
FUCAPE & 04 & 02 & USCS & 01 & UNIFAVIP & 00 & 00 \\
UNIVALI & 02 & 01 & F.N.H & 01 & PUC - CAMP. & 00 & 00 \\
FAT & 01 & UNEMAT & 01 & UFAM & 00 & 00 \\
UNIFRA & 01 & UFRRJ & 01 & PUCRJ & 00 & 00 \\
\hline
\end{tabular}

Nota: Frequência - F.; Universidad Santo Tomás - UST; Universidad de Valencia - UV; Univ. of Arkansas - UARK; Faculdade Maurício de Nassau - UNINASSAU; University of Bologna - UNIBO; PUC - Campinas; Faculdade Novos Horizontes - F.N.H.; Una-Puno - Peru - U.P.P.; University of Roehampton - U.R.

Fonte: Dados da pesquisa (2015).

Segundo Maia e Caregnato (2008) um dos principais pontos a serem analisados em uma estrutura de rede refere-se à posição ocupada pelo ator em relação a toda estrutura de relacionamento entre os membros que participam de uma rede. Estes autores relatam que a posição de centralidade está associada ao poder exercido pelo o ator na rede, o que implica em ter menos restrições e mais oportunidades nas relações com os demais membros dessa estrutura.
Mizruchi (2006) relata que a maioria dos estudos sobre a relação entre centralidade e poder condizem com os pressupostos de que a posição de um agente numa estrutura social tem impacto significativo sobre seu comportamento e bem-estar. 0 autor enfatiza três áreas relevantes na análise de redes sociais: os efeitos da centralidade do agente sobre o comportamento, a identificação de subgrupos e a natureza das relações entre as organizações. 
As medidas do grau de centralização buscam indicar o quanto importante é um ator nas relações entre os membros de um grupo. 0 Grau de intermediação (betweenness centrality) refere-se à quantidade de vezes que um determinado ator é utilizado por outro, como caminho para alcançar um terceiro individuo. $\mathrm{Na}$ análise do grau de centralidade e interações entre as instituições na formação de redes sociais do congresso ANPCONT, a USP apresenta um papel central nos canais de articulação e fluxo de interações entre os atores dessa rede com $38,6 \%$. As instituições UNISINOS com $16.5 \%$, e UFPB com $11.4 \%$, também se destacam, seguidas pela UFPR com $9.88 \%$ e FURB com $7.57 \%$ ambas com $0.09 \%$ de centralização (Tabela 2).

\begin{tabular}{|c|c|c|c|c|c|c|c|c|}
\hline INSTITUIÇÃO & G.C. & G.I. & INSTITUIÇÃO & G.C. & G.I. & INSTITUIÇÃO & G.C. & G.I. \\
\hline USP & 0.195 & 38.692 & UFSM & 0.024 & 0.000 & PUCPR & 0.012 & 0.000 \\
\hline UNISINOS & 0.110 & 16.561 & UNIPAMPA & 0.024 & 0.000 & ESADE & 0.012 & 0.000 \\
\hline UFPB & 0.085 & 11.494 & ESTACIO & 0.024 & 0.000 & ISPA & 0.012 & 0.000 \\
\hline UFPR & 0.098 & 9.884 & CEFET/MG & 0.012 & 0.000 & FAED & 0.012 & 0.000 \\
\hline FURB & 0.098 & 7.571 & UNEB & 0.012 & 0.000 & UFERSA & 0.012 & 0.000 \\
\hline MACKENZIE & 0.061 & 6.188 & UNIFOR & 0.012 & 0.000 & IBMEC & 0.012 & 0.000 \\
\hline UFRJ & 0.061 & 5.897 & UCB & 0.012 & 0.000 & IMED & 0.012 & 0.000 \\
\hline UnB/UFPB/UFRN & 0.073 & 5.345 & ITCP & 0.012 & 0.000 & UARK & 0.012 & 0.000 \\
\hline UFMG & 0.061 & 5.330 & UFF & 0.012 & 0.000 & UEMS & 0.012 & 0.000 \\
\hline UFU & 0.049 & 5.330 & UFPE & 0.012 & 0.000 & UDESC & 0.012 & 0.000 \\
\hline FGV & 0.073 & 4.618 & FAESA & 0.012 & 0.000 & U.S.T. & 0.000 & 0.000 \\
\hline UERJ & 0.037 & 3.583 & U.P.P. & 0.012 & 0.000 & U.V. & 0.000 & 0.000 \\
\hline UFRGS & 0.024 & 3.553 & FURG & 0.012 & 0.000 & FECAP & 0.000 & 0.000 \\
\hline UFBA & 0.024 & 3.553 & FAMETRO & 0.012 & 0.000 & UEPB & 0.000 & 0.000 \\
\hline UFSC & 0.049 & 1.925 & UEM & 0.012 & 0.000 & PUC MINAS & 0.000 & 0.000 \\
\hline FUCAPE & 0.049 & 1.842 & USCS & 0.012 & 0.000 & UNIFAVIP & 0.000 & 0.000 \\
\hline UNIVALI & 0.024 & 1.807 & F.N.H & 0.012 & 0.000 & PUC - CAMP. & 0.000 & 0.000 \\
\hline FAT & 0.024 & 1.807 & UNEMAT & 0.012 & 0.000 & UFAM & 0.000 & 0.000 \\
\hline UNIFRA & 0.024 & 1.807 & UFRRJ & 0.012 & 0.000 & PUCRJ & 0.000 & 0.000 \\
\hline SJT & 0.024 & 1.807 & UNOESC & 0.012 & 0.000 & UFSCAR & 0.000 & 0.000 \\
\hline UFES & 0.049 & 1.703 & UNICAMP & 0.012 & 0.000 & UFRPE & 0.000 & 0.000 \\
\hline UNB & 0.061 & 1.079 & UNESC & 0.012 & 0.000 & UNISUL & 0.000 & 0.000 \\
\hline
\end{tabular}




\begin{tabular}{lcclccccc}
\hline \multicolumn{1}{c}{ INSTITUIÇÃO } & G.C. & G.I. & INSTITUIÇÃO & G.C. & G.I. & INSTITUIÇÃO & G.C. & G.I. \\
\hline UFC & 0.037 & 0.522 & UCS & 0.012 & 0.000 & UNESPAR & 0.000 & 0.000 \\
UFRN & 0.037 & 0.231 & UNOCHAPECÓ & 0.012 & 0.000 & UFPA & 0.000 & 0.000 \\
UEL & 0.024 & 0.181 & UNINASSAU & 0.012 & 0.000 & IFPB & 0.000 & 0.000 \\
UNIBO & 0.012 & 0.151 & UTFPR & 0.012 & 0.000 & UPM & 0.000 & 0.000 \\
UFG & 0.024 & 0.000 & FAP & 0.012 & 0.000 & & & \\
U.R. & 0.024 & 0.000 & UNIOESTE & 0.012 & 0.000 & & & \\
\hline
\end{tabular}

Nota: G.C - Grau de centralidade, G.I - Grau de intermediação.

Fonte: Dados da pesquisa (2015)

A centralidade de um ator não é uma posição fixa, nem hierárquica, mas pode ser traduzida como poder. A posição central de uma pessoa na rede favorece a troca de informação e comunicação entre os atores desse grupo. No entanto, o fato do indivíduo estar ou não em uma posição central, não significa necessariamente que este ator, não esteja bem posicionado, em um sistema de inter-relações na rede social a que este integra.

A rede formada nas inter-relações da produção científica entre as instituições do Congresso ANPCONT, apresenta artigos com um ou mais autores. Nesse cenário, a análise desse estudo refere-se ao número de colaborações entre instituições participantes com apenas um autor ou diversos.

Newman (2004) destaca que, a rede de coautoria formada na comunidade acadêmica, representa um excelente recurso para a prossecução de questões estruturais que acaba por revelar muitas características e mudanças nos padrões de colaboração ao longo do tempo, bem como na disseminação do conhecimento nas relações entre os atores dessa rede.

Estudos de rede social geralmente utilizam a abordagem matemática da teoria dos grafos, que oferece uma linguagem formal para descrever as características de uma rede social. Esta teoria proporciona uma tradução dos dados de uma matriz em conceitos formais e teoremas, que podem ser diretamente relacionados com as características substantivas nas relações entre os atores. Embora a teoria dos grafos não seja a única teoria matemática utilizada para modelar redes sociais, de forma geral, é considerada como um ponto de partida para a análise de redes sociais.

A reprodução dos dados da matriz relacional é apresentada por meio de sociogramas. 0 reconhecimento de que os sociogramas poderiam ser utilizados para estudar a estrutura social, levou a uma rápida introdução dessas técnicas analíticas nos estudos de redes sociais (SCOTT, 2000; WASSERMAN; FAST, 1994).

Para Katz e outros autores (2004) o sociograma é uma apresentação visual de todos os laços e nós de uma rede, a qual mostra se há muitas ou poucas ligações entre os membros de uma organização. Considerando que esta visualização permite identificar padrões e localizar onde cada indiv[iduo está situado na rede, o sociograma pode fornecer uma visão geral da relação entre os membros dessa organização.

Pesquisadores utilizam uma variedade de métricas para quantificar diferenças importantes na estrutura de uma rede. As Métricas mais utilizadas incluem grau de centralidade (atores enviam ou recebem ligações diretas), grau de intermediação (atores têm laços 
com outras pessoas que não estão diretamente ligados), grau de proximidade (atores estão direta ou indiretamente ligado ao resto na rede), reciprocidade (relações mútuas entre atores), e transitividade (medida em que os agentes que estão ligados uns aos outros também estão ligados a outros agentes).

Nesse contexto, a sociometria caracteriza-se como um método que investiga a organização de grupos sociais, sua evolução e a posição que todos os indivíduos ocupam nesse grupo qualificado como rede. Essa metodologia busca compreender a estrutura de um grupo, e sua principal preocupação é com as inter-relações formadas pelos membros dessa rede. As análises lidam com o estudo matemático de propriedades psicológicas nas populações por meio de aplicação de métodos quantitativos, que permitem visualizar características semelhantes entres os indivíduos de uma estrutura organizacional (MORENO, 1934).
No sociograma, a seguir, é apresentada a rede de coautorias entre os pesquisadores das instituições, objeto deste estudo (Figura 2). As redes sociais formadas entre as instituições na produção de publicações científicas com grau de centralidade "entrada/saída" mais elevado foram observadas entre as instituições USP, UNISINOS, UFPB. Entretanto, algumas instituições UFPR, FURB, MACKENZIE, UFRJ, UnB/UFPB/UFRN, UFMG e UFU também se destacaram por apresentar laços e interações com outras unidades do grupo.

O sociograma demonstra a rede social formada entre as instituições, e os nodos (conjunto de pontos) ligados por linhas (arcos), que se removidas desconectam a rede que compõe a amostra nas relações entre os atores deste estudo. A representação por meio do sociograma permite visualizar que quanto maior a representação gráfica do nodo, maior sua importância para a manutenção da rede.

Figura 2 - Redes Sociais de cooperação entre Instituições

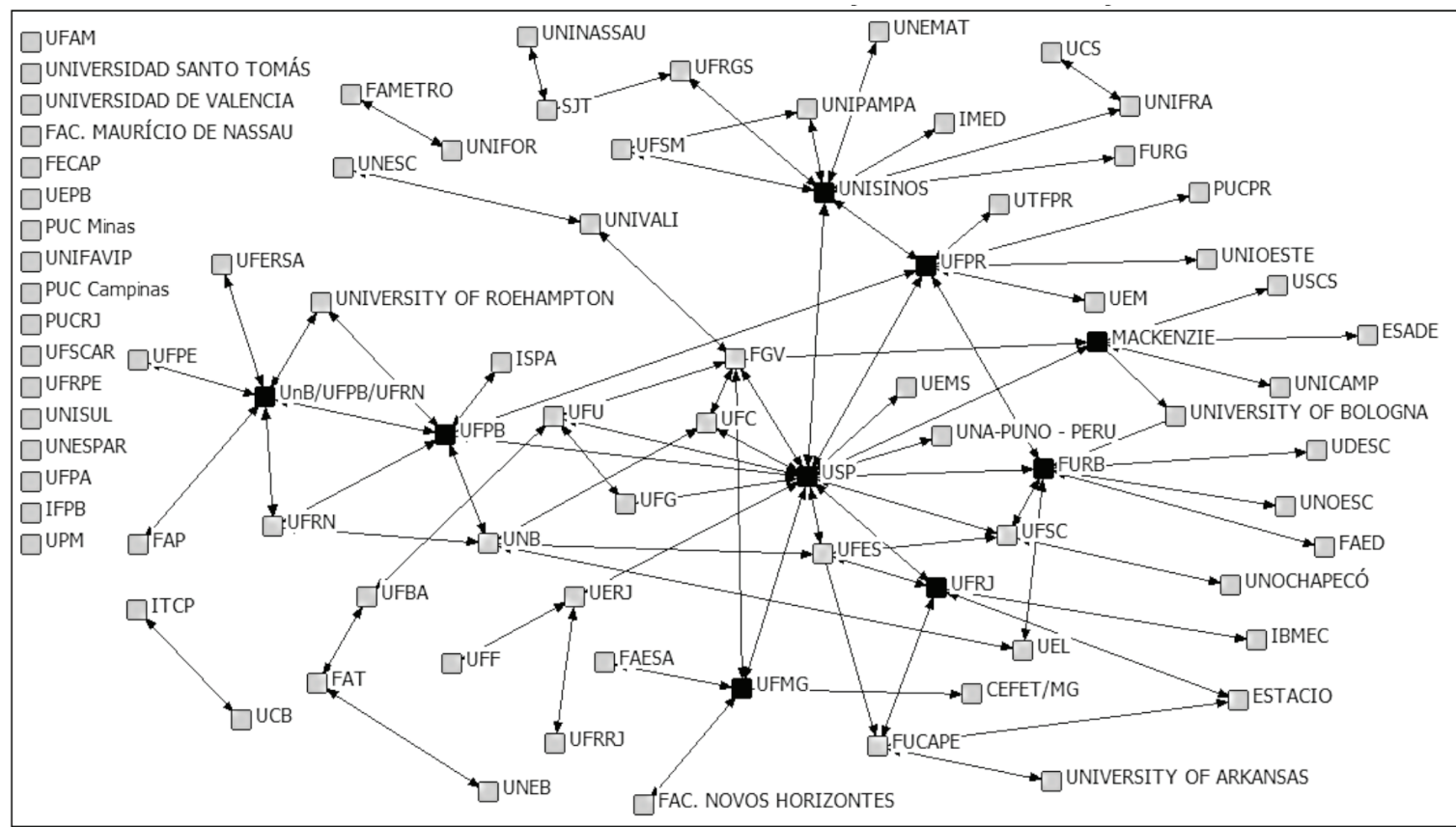

Fonte: Dados da pesquisa (2015). 
A análise de rede social é feita a partir de pressupostos que ajudam a entender a dinâmicas e as interações nas relações entre indivíduos ou grupos que compõe uma rede. A análise das interações institucionais e autorias já foi objeto de outros estudos similares, que tem como premissas as inter-relações na formação de redes sociais, tais como: Guimarães e outros autores (2009), Balestrin, Verschoore e Reyes (2010), Nascimento e Beuren (2011), e Ribeiro (2012).

Guimarães e outros autores (2009) realizaram uma análise das relações acadêmicas e atributos com ênfase na formação de redes sociais nos programas de pós-graduação em administração do Brasil. Os resultados desse estudo demonstraram que o programa da UFRGS apresenta-se como o mais central e um dos quatro mais prestigiados nas relações entre os atores, seguido pelos programas da FGV/SP, USP/SP, UFRGS e FGV/RJ. Além disso, os autores concluíram que os relacionamentos entre os programas são esparsos e na maioria das vezes fracos.

Balestrin, Verschoore e Reyes (2010) estudaram as características no campo das redes de cooperação interorganizacionais no Brasil, na área de estudos organizacionais. A análise bibliométrica desse estudo demonstrou que, três instituições apresentaram grau de centralidade elevado, em se tratando especificamente dos possíveis laços relacionais que unem duas ou mais instituições nas publicações, sendo estas: a UFRGS a UFRJ e a UNISINOS.

Nascimento e Beuren (2011), por meio de uma visão panorâmica na formação de redes sociais mapearam a produção científica dos programas de pós-graduação de ciências contábeis do Brasil, no período entre 2007 a 2009, utilizando a metodologia bibliométrica e sociométrica. Neste estudo, as interações mostraram-se fracas e esparsas e os autores identificaram como principal ator nos laços da rede social a USP, seguida pela UPM, USP-RP, UFMG, FUCAPE, UNIFECAP e a FURB respectivamente.
Ribeiro (2012) examinou a produção acadêmica da Revista Contemporânea de Contabilidade no período entre 2004 a 2012, sob a concepção de parceria formada entre instituições e os autores mais profícuos. Os principais resultados deste estudo constataram um maior grau de centralidade e intermediação para as Instituições de ensino superior UFSC e USP.

Segundo Freitas (2008), a representação gráfica permite explorar as informações subjacentes a análise de redes sociais, realizadas por meio da visualização dessa rede, em grafos, onde os nodos representam os atores dessa rede (pessoas, instituições, grupos etc.) e as arestas, o relacionamento nas relações entre componentes centrais ou periféricos de interações entre os membros da rede.

As evidências observadas nesta pesquisa corroboram com os estudos de Guimarães e outros autores (2009), Balestrin, Verschoore e Reyes (2010), Nascimento e Beuren (2011) e Ribeiro (2012), que destacam os benefícios na formação de redes sociais entre instituições e a necessidade em fortalecer laços e partilha de informações para disseminação de conhecimentos. Neste estudo foram registradas produções de artigos em díades, tríades e individuais (17 nós soltos). Os artigos individuais caracterizam uma lacuna estrutural por não estabelecer relações com outras instituições. No entanto, a produção individual ou em coautorias muitas vezes, é influenciada pela especificidade do campo do conhecimento e na forma de produção.

O uso das redes sociais na análise da produção científica permite a observação de aspectos interdisciplinares ocasionados por meio da cooperação entre os pesquisadores, além de proporcionar a análise e estruturação de um dado campo do conhecimento (NASCIMENTO; BEUREN, 2011). Burt (1992) relata que as redes sociais permitem trocas de informações entre os atores que a compõem e o ambiente onde os atores estão inseridos. Maciel (2007) descreve a importância dos relacionamentos interorganizacionais, assim como as ligações derivadas, tanto de dependência como de 
interdependência dos pesquisadores de redes, prevalecendo o interesse pela troca de informações.

\section{CONSIDERAÇ̃̃ES FINAIS}

A produção científica que aborda a temática rede social vem crescendo exponencialmente nas últimas décadas; dada a sua importância, pesquisadores, acadêmicos e o público em geral de diversas áreas, utilizam essa ferramenta como canal comunicação entre colaboradores. Este estudo teve como objetivo verificar quais as redes sociais institucionais formadas nos estudos apresentados no Congresso ANPCONT no período entre 2013 a 2015. A população da pesquisa abrangeu 334 artigos publicados no congresso ANPCONT. A amostra final foi composta de 165 artigos com 17 produções individuais e 148 coautorias em grupos interligados na produção cientifica.

Os resultados desta pesquisa indicam que, as instituições, mais profícuas foram a USP, UNISINOS, UFPR e FURB em número de artigos apresentados no Congresso ANPCONT.

A análise realizada por meio de grafo constatou a formação de uma rede de cooperação envolvendo dez instituições: UFPR, FURB, MACKENZIE, UFRJ, UnB/ UFPB/UFRN, UFMG, UFU e UFGV, que se destacaram por apresentar interações com outras unidades do grupo, além das mencionadas anteriormente. Observa-se também, laços centrais "entrada/saída" na criação de grupo interligado composto pelas instituições do programa UnB/UFPB/UFRN e FGV, que apresenta grau de centralidade elevado correspondendo a 0.073 , em ambos os casos e interações significativas com outros atores dessa rede.

Considerando os benefícios na formação de rede sociais e as inter-relações entre os atores, observou-se nesse estudo a formação de grupos dispersos. Concluímos que, a rede de cooperação e produção científica entre as instituições apresentam ligações fracas e conexões esparsas.

Os resultados desse estudo apóiam os resultados de outras pesquisas com abordagem temática em redes sociais, tais como: Guimarães e outros autores (2009), Balestrin, Verschoore e Reyes (2010), Nascimento e Beuren, (2011) e Ribeiro (2012), cujo as premissas na formação de laços institucionais, promovem a partilha e intercâmbio de informações na produção acadêmica.

Sugere-se para futuras pesquisas: (a) aplicar o mesmo estudo em outros congressos ou reuniões científicas nas áreas de Ciências Contábeis ou correlatas; (b) analises comparativas de outros temas entre as instituições, (c) Verificar a formação de redes sociais nas produções acadêmicas de coautorias entre estudantes de outras instituições.

\section{REFERÊNCIAS}

AZEVEDO, T.B.; RODRIGUES, DR. M.V.R. Softwares para Análise de Redes Sociais - ARS. In: Congresso Nacional de Excelência em Gestão, 6, 2010, Rio de Janeiro. Anais..., Rio de Janeiro, 2010. p. 19.

BALESTRIN, A.; VERSCHOORE, J.R.; REYES JR., E. O campo de estudo sobre redes de cooperação interorganizacional no Brasil. Revista de Administração

Contemporânea, v.14, n.3, 2010. p.458-477.

\section{BARABÁSI, A.L. Linked. How Everything is} Connected to Everything else and What it means for Business, Science and Everydai Life. Cambridge: Plume, 2003.

BASTOS, V.B. SANTOS, M.V. Redes informais e compartilhamento de significados sobre mudança organizacional. RAE - Revista de Administração de Empresas, v.47, n.3, São Paulo, 2007. p.27-39. 
BRITTO, J. Redes de cooperação entre empresas. Estrutura de mercado e inovação. In: KUPFER, D.; HASENCLEVER, L. Economia Industrial: fundamentos teóricos e práticas no Brasil. Rio de Janeiro: Campos. 2002.

BRUFEN, L.S.R.F; GABRIEL JR.; SORRIBAS, T.V. Redes sociais na pesquisa científica da área de ciência da informação. DataGramaZero - Revista de Informação, v.12 n.3, ago. 2011.

BURT, R.S. Structural holes: the social structure of competition. Cambridge: Harvard University Press, 1992.

BUCHANAN, M. Nexus: Small Worlds and the Groundbreaking Theory of Networks. New York: W.W. Norton e Company, 2002.

CARTER, C.R.; ELLRAM, L.M.; TATE, W. The use of Social Network Analysis in logistics research. Journal of Business Logistics, v.28, n.1, 2007. p.137-168.

DEGENNE, A.; FORSÉ, M. Introducing Social Networks. London: Sage, 1999.

FREITAS, C.M.D.S. et al. Extração de conhecimento e análise visual de redes sociais. In: SEMISH -

Seminário Integrado de Software e Hardware, Belém do Pará, Brasil, SBC, 2008. p.106-120.

GRANOVETTER, M. The Strenght of Weak Ties. American Journal of Sociology, v.78, 1973. p.1360-1380.

GRANOVETTER, M. The strength of weak ties: a network theory revisited. Social Theory, v.1, 1983. p.201-233.

GUIMARÃES, T.A. et al. A rede de programas de pós-graduação em administração no Brasil: analise de relações acadêmicas e atributos de programas.

Revista de Administração Contemporânea, v.13, n.4, 2009. p.564-582.
HANNEMAN, R.; RIDLLE, M. Introduction to social network methods. Riverside: University of California. 2005.

KATZ, N.; LAZER, D.; ARROW, H.; CONTRACTOR, N. Network theory and small groups. Small. Group

Research, v.35, n.3, 2004. p.307-332.

LOIOLA, E.; MOURA. S. Análise de redes: uma contribuição aos estudos organizacionais. In: FISCHER, T. Gestão contemporânea: cidades estratégicas e organizações locais. Rio de Janeiro: FGV, 1996. p.53-68.

LOZARES COLINA, C. La teoria de redes sociales. Papers n.48, Universidad Autònoma de Barcelona, 1996.

MACIAS-CHAPULA, C.A. O papel da informetria e da cienciometria e sua perspectiva nacional e internacional. Ciência da Informação, v.27, n.2, 1998. p.64-68.

MACIEL, C.O. Práxis estratégicas e imersão social em uma rede de organizações religiosas. 2007; Dissertação (Mestrado) - Universidade Federal do Paraná, Curitiba-PR, Brasil, 2007.

MARTELETO, R.M.; SILVA; ANTÔNIO, B.O. Redes e capital social: o enfoque da informação para o desenvolvimento local. Ci. Inf., Brasília, v.33, n.3, set-dez. 2004. p.41-49.

MARTELETO, R.M.; SILVA; ANTÔNIO, B.O. Redes sociais, mediação e apropriação de informações: situando campos, objetos e conceitos na pesquisa em Ciência da Informação. Tendências da Pesquisa Brasileira em Ciência da Informação, v.3, n.1, João Pessoa, 2010. p.27-46;

MARTINS, G.A.; THEÓPHILO, C.R. Metodologia da investigação científica para ciências sociais aplicadas. São Paulo: Atlas, 2007. 
MARTINS, G.S. A construção do conhecimento científico no campo de gestão de operações no Brasil: uma análise sob a ótica de redes sociais do período 1997-2008. 2009; Dissertação (Mestrado) Fundação Getúlio Vargas, São Paulo-SP, Brasil, 2009.

MIZRUCHI, M.S. Análise de redes sociais: avanços recentes e controvérsias atuais. Revista de Administração de Empresas, v.46, n.3, 2006. p.10-15.

MORENO, J.L. Who shall survive? A new approach to the problem of human interrelations. Washington, DC: Nervous and Mental Disease Publishing Company, 1934.

NASCIMENTO, S.; BEUREN, I.M. Redes Sociais na Produção Científica dos Programas de PósGraduação de Ciências Contábeis do Brasil. Revista de Administração Contemporânea, v.15, n.1, jan-fev. 2011. p.47-66.

NEWMAN, M.E.J. Coauthorship networks and patterns of scientific collaboration. Proceedings of the National Academy of Sciences of the United States of America, v.101, n.1, Washington, apr. 2004. p.5200-5205.

NELSON, R. O uso da Análise de Redes Sócias no estudo das estruturas organizacionais. Revista de Administração de Empresas, v.24, n.4, 1984. p.150-157.

OTTE, E.; ROUSSEAU, R. Social network analysis: a powerful strategy, also for the information sciences. Journal of Information Science, v.28, n.6, 2002. p.441-453.
RECUERO, R. Redes Sociais na Internet:

Considerações Iniciais. E Compós, v.2, 2005.

RIBEIRO, H.C.M. Uma análise do perfil da produção acadêmica durante o período de 2004 a 2012.

Revista Contemporânea de Contabilidade, v.10, n.20, 2013. p.3-28.

ROSSONI, L.; HOCAYEN-DA-SILVA, A. J.; JÚNIOR, I. F. Aspectos estruturais da cooperação entre pesquisadores no campo de administração pública e gestão social: análise das redes entre instituições no Brasil. Revista de Administração Pública, v.42, n.6, 2008. p.1041-1067.

SCOTT, J. Social Network Analysis: a handbook. 2.ed. London: Sage Publications, 2000.

SILVA, A.B.O. et al. Análise de redes sociais como metodologia de apoio para a discussão da interdisciplinaridade na ciência da informação. Ciência da Informação, v.35, n.1, 2006. p.72-93.

WASSERMAN, S.; FAUST, K. Social network analysis: methods and applications. Cambridge: Cambridge University Press, 1994.

WATTS, D. J. Six Degrees. The Science of a Connected Age. New York: W. W. Norton \& Company, 2003.

WELLMAN, B. Networks in the Global Village: Life in Contemporary Communities, Boulder, Colorado: Westview Press, 1999. p.331-366. 
Recebido em: 10 de março de 2016

Avaliado em: 5 de junho de 2016

Aceito em: 26 de julho de 2016
1. Mestre em Contabilidade pela Universidade Federal do Paraná - UFPR.

E-mail: marcianzilago@gmail.com

2. Mestre em Contabilidade pela Universidade Federal do Paraná - UFPR.

E-mail: f.j.melo@uol.com.br 